\title{
Cell adhesion of Shewanella oneidensis to iron oxide minerals: Effect of different single crystal faces
}

\author{
Andrew L. Neal ${ }^{\text {a) }}$ \\ Savannah River Ecology Laboratory, University of Georgia, Aiken, South Carolina 29808 \\ Tracy L. Bank ${ }^{\text {b) }}$ and Michael F. Hochella, Jr. \\ Department of Geosciences, Virginia Polytechnic Institute and State University, Blacksburg, \\ Virginia 24060

\begin{abstract}
Kevin M. Rosso
The W.R. Wiley Environmental Molecular Sciences Laboratory, Pacific Northwest National Laboratory, Richland, Washington 99352
\end{abstract}

(Received 25 July 2005; accepted 22 November 2005; published 30 December 2005)

\begin{abstract}
The results of experiments designed to test the hypothesis that near-surface molecular structure of iron oxide minerals influences adhesion of dissimilatory iron reducing bacteria are presented. These experiments involved the measurement, using atomic force microscopy, of interaction forces generated between Shewanella oneidensis MR-1 cells and single crystal growth faces of iron oxide minerals. Significantly different adhesive force was measured between cells and the (001) face of hematite, and the (100) and (111) faces of magnetite. A role for electrostatic interactions is apparent. The trend in relative forces of adhesion generated at the mineral surfaces is in agreement with predicted ferric site densities published previously. These results suggest that near-surface structure does indeed influence initial cell attachment to iron oxide surfaces; whether this is mediated via specific cell surface-mineral surface interactions or by more general interfacial phenomena remains untested. (C) 2005 American Institute of Physics. [DOI: 10.1063/1.2151110]
\end{abstract}

\section{INTRODUCTION}

Sufficient energy is available from the reduction of ferric iron in both crystalline ${ }^{1}$ and noncrystalline ${ }^{2}$ iron oxides to support the growth of dissimilatory metal reducing bacteria where ferric iron is the dominant electron acceptor. It is now clear that the relative reactivity of various oxide phases in both terrestrial and marine sediments is controlled less by thermodynamic considerations than the surface area of the respective phases. ${ }^{1,3,4}$ In instances where direct cell-mineral contact is necessary for bacterial iron reduction, ${ }^{5-7}$ mineral surface area controls not only the availability of reactive sites but also of cell attachment sites. Ferrous iron may significantly limit bioreduction by occluding reactive sites thereby controlling the observed reactivity. ${ }^{8,9}$ It has been recently demonstrated that when mineral surface area is controlled by presenting cells with single crystal growth faces, differences in cell accumulation of the dissimilatory iron reducing bacterium Shewanella oneidensis MR-1 are observed between three iron oxide faces which moreover appear consistent with modeled rates of electron transfer from an outer membrane cytochrome. ${ }^{10}$ Thus, fine scale interactions reflecting the atomic or electronic structure of Fe-oxide bulk and nearsurface regions also have the potential to affect biological activity/reduction.

At present we have only a very general understanding of how dissimilatory iron reducing bacteria (DIRB) attach to iron oxide surfaces: hydrophobic interactions are implicated

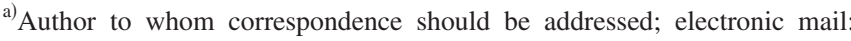
neal@srel.edu

b) Present address: Oak Ridge National Laboratory, Oak Ridge, TN 37831.
}

in the adhesion of Shewanella algae BrY to iron oxides, ${ }^{11}$ this hydrophobicity may be expressed via a proteinaceous component since cell adhesion is interrupted by proteases. ${ }^{12}$ In this sense, adhesion of DIRB is similar to adhesion of a wide variety of bacteria to inanimate surfaces. ${ }^{13-15}$ However, since DIRB are reliant upon electron transfer to iron oxide surfaces when growing anaerobically, there exists the potential for specific adhesion.

Here we describe force measurements, made using atomic force microscopy (AFM) and S. oneidensis MR-1 cells immobilized onto tip-less silicon nitride cantilever apices, to a series of flat single crystal iron oxide faces. In a series of similar experiments using a variety of bacterial cells, including $S$. oneidensis, Lower and colleagues have sought to improve the technique of immobilizing cells at a cantilever surface so that they are not only alive, but also limited in number, allowing more reliable measurements to be performed. ${ }^{16-18}$ Their approach was to immobilize cells employing either poly $(L$-lysine) treated glass beads, subsequently attached to the cantilever with epoxy resin, ${ }^{17}$ or to attach individual cells to cantilever surfaces previously treated either with poly( $L$-lysine) or amine functionalized with an aminosilane. ${ }^{18}$ The surface pretreatment promotes cell adhesion to the glass-cantilever surface. Using these approaches, Lower and colleagues established two paradigms describing the interaction of DIRB with mineral surfaces: $:^{18}$ based upon increased forces of adhesion measured on goethite surfaces compared to diaspore, they established that specific interactions are elicited between DIRB and iron oxide surfaces; second, they interpreted retraction curves collected on goethite displaying "sawtoothlike discontinuities" 
as bond failure events, indicative of specific interactions between the iron oxide surface and outer membrane associated cytochromes. This interaction was successfully modeled using a wormlike chain model to mimic successive bond failure between the outer membrane associated protein and the mineral surface.

The use of single crystal surfaces minimises differences in surface area between the three faces limiting possible effects of surface topography which can significantly affect forces of bacterial adhesion to surfaces. ${ }^{19,20}$ The surfaces we employed have limited surface roughness as determined by AFM $(<1 \mathrm{~nm}$ rms $)$. We demonstrate that the adhesion forces measured at the three faces are significantly different and support previous observations that hematite presents a more optimal surface for cell activity than magnetite surfaces. The data suggests that electrostatic interactions are potential determinants of cell adhesion and need to be considered along with rates of electron transfer when considering the relative reactivities of different iron oxides towards dissimilatory iron reduction.

\section{MATERIALS AND METHODS}

Iron oxide minerals: Natural, single crystal growth faces of iron oxide minerals were used in these experiments (surfaces are described in Ref. 10). Hematite (001) faces were isolated on specular, tabular hematite from Brazil acquired from the Stanford Mineralogical Collection (sample 51080). Magnetite (100) faces were isolated on tetrahexahedra from New York, acquired from the Harvard Mineralogical Collection (sample 134816). Magnetite (111) faces were isolated on octahedra from Vermont, again from the Harvard Mineralogical Collection (sample 93.1.5.2). We used natural crystal growth faces for this study. Samples were previously determined by low resolution x-ray photoelectron spectroscopy (XPS) to be free of surface contaminants, within the sensitivity limits of XPS, ${ }^{10}$ however, surfaces were not cleaned other than by rinsing in distilled deionized water to remove nonadherent material. The aim of this research was to determine cell-mineral interactions under near natural conditions. Since mineral surfaces are likely to be covered with adventitious carbon under natural conditions we made no attempt to subject the mineral surface to chemical treatment which might not only remove adventitious material but also significantly influence the near surface physicochemistry of the three mineral faces. Previously determined XPS iron-oxygen and divalent and/or trivalent iron ratios were consistent with the expected bulk stoichiometries of hematite and magnetite. We have thus assumed that any adventitious carbonaceous layers present are qualitatively similar across the three mineral faces and arise either from prior storage of the surfaces or from exposure to the PIPES-buffered medium in which AFM measurements were performed. Atomic force microscopy (AFM) was used to assess surface topography (see Ref. 10). Surfaces used possessed a mean square roughness of $\leqslant 1 \mathrm{~nm}$ over lateral areas of several square microns and were optically flat and "mirrorlike."

Cell culture: Shewanella oneidensis MR-1 was cultured anaerobically in a defined salt medium $(\mathrm{SM}) .^{21}$ Besides
$32 \mathrm{mM}$ lactate which serves both as a carbon source and electron donor, SM contains $32 \mathrm{mM}$ PIPES buffer, $9 \mathrm{mM}$

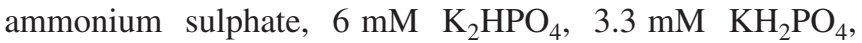
$2 \mathrm{mM} \mathrm{NaHCO}_{3}, 1 \mathrm{mM} \mathrm{MgSO}_{4}, 0.5 \mathrm{mM} \mathrm{CaCl}_{2}, 67.2 \mu \mathrm{M}$ $\mathrm{Na}_{2}$-EDTA, 56.6 $\mu \mathrm{M} \mathrm{H}_{3} \mathrm{BO}_{3}, 10 \mu \mathrm{M} \mathrm{NaCl}, 5.4 \mu \mathrm{M} \mathrm{FeSO}_{4}$, $5 \mu \mathrm{M} \mathrm{CoSO}, 5 \mu \mathrm{M} \quad \mathrm{Ni}\left(\mathrm{NH}_{4}\right)_{2} \mathrm{SO}_{4}, 3.9 \mu \mathrm{M} \quad \mathrm{Na}_{2} \mathrm{MoO}_{4}$, $1.5 \mu \mathrm{M} \quad \mathrm{Na}_{2} \mathrm{SeO}_{4}, \quad 1.3 \mu \mathrm{M} \quad \mathrm{MnSO}_{4}, \quad 0.2 \mu \mathrm{M} \quad \mathrm{CuSO}_{4}$, $20 \mathrm{mg} \mathrm{L}^{-1}$ each of arginine, serine and glutamic acid. Cells were grown anaerobically for 24 hours in batch culture with $32 \mathrm{mM}$ fumerate as electron acceptor. We chose an organic terminal electron acceptor to prevent the formation of metal precipitates on the cell surface (likely to form with either $\mathrm{Fe}^{3+}$ - or $\mathrm{Mn}^{4+}$-containing electron acceptors) which would preclude the study of true cell-surface, mineral-surface interactions. However, since phenotypic differences are well documented for cells grown under different electron acceptor availabilities, fumerate was chosen instead of other organic electron acceptors (i.e., $\mathrm{O}_{2}$ or $\mathrm{NO}_{3}$ ) since, when compared to cells grown on $\mathrm{Fe}^{3+}$ as terminal electron acceptor, cells grown on fumerate are phenotypically less distinct than cells grown on either $\mathrm{O}_{2}$ or $\mathrm{NO}_{3} \cdot{ }^{22}$ All cells were cultured in anaerobic flasks using butyl rubber septa and aluminium crimp caps (Wheaton, Millville, NJ). Cells were harvested by centrifugation under a $95 \% \mathrm{~N}_{2}: 5 \% \mathrm{H}_{2}$ atmosphere and washed in an anaerobic basal medium lacking both lactate and fumerate. Cells were washed three times and resuspended in a final volume of $1 \mathrm{ml}$ in anaerobic basal medium.

Immobilization of cells onto cantilever apices: To maximize cell adhesion while maintaining cell viability, tipless $\mathrm{Si}_{3} \mathrm{~N}_{4}$ cantilever (Veeco, Chadds Ford, PA) surfaces were functionalized using 3-aminopropyltriethoxysilane (APTES, Sigma, A-3648), generating a surface coating of aminosilane groups. Shewanella cells readily attach to cantilevers treated in this way and the strength of the cell-cantilever interaction is of sufficient magnitude to prevent cell detachment during experimentation at iron oxide surfaces. ${ }^{17,18} \mathrm{Can}-$ tilevers were soaked in an APTES solution (2\% in $95 \%$ aqueous acetone) at room temperature for 15 minutes before being rinsed exhaustively in acetone. Silane linkages were cured at $110{ }^{\circ} \mathrm{C}$ for 1 hour. Cells were attached to the end of the APTES-treated cantilevers by lowering cantilevers onto dilute solutions of $S$. oneidensis, allowing one or two cells to attach at the apex of the cantilever. Since it was imperative to limit cell exposure to oxygen, attached cells were briefly visualized prior to experimentation with light microscopy to verify the position and number of cells on the cantilevers. Confirmation of the number and position of cells at the cantilever surface was obtained following experimentation using confocal scanning laser microscopy (see Fig. 1). In total, three cantilevers with attached cells were used in these experiments. A statistical treatment was used to account for differences in force measurements between cantilevers which might be due to not only the number and position of cells, but also differences in cell metabolic activity. Each cantilever was used to make measurements on each crystal face, thus creating nine data sets. The order in which measurements were collected on the three mineral faces was different for each cantilever, eliminating bias in adhesion measurements which may reflect more the order in which measure- 


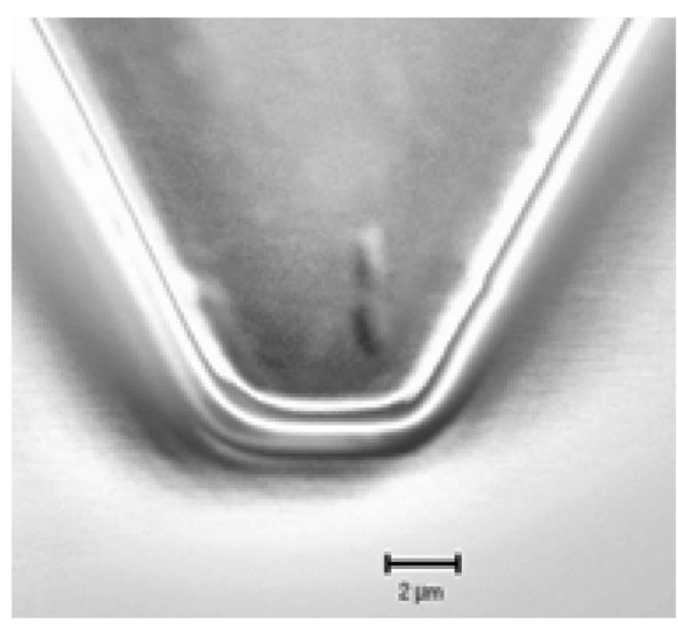

FIG. 1. Confocal scanning laser micrograph of Shewanella oneidensis MR-1 cells attached to the surface of an APTES-functionalized tipless cantilever. Cantilever was imaged after force measurements were performed. Scale bar indicates $2 \mu \mathrm{m}$.

ments were taken on the mineral surfaces rather than inherent properties of the mineral surfaces themselves. Cantilevers were considered as a factor in two-factor analyses of variance, the other factor (and main factor of interest) being crystal face.

Atomic force microscopy measurements: AFM experiments were conducted in contact mode using a Nanoscope IIIa multimode SPM (Digital Instruments, Santa Barbara, $\mathrm{CA}$ ) at room temperature. The AFM measurements were performed in a fluid cell containing anaerobic PIPES bufferbased medium (32 $\mathrm{mM}$ ) comparable to that used in previous growth experiments ${ }^{10}$ except lacking in a carbon source (lactate). The $\mathrm{pH}$ of the solution was thereby maintained at 7 . Under these conditions MR-1 cells have a zeta $(\zeta)$ potential of $-5.7 \pm 0.5 \mathrm{mV}$ (Zetasizer $3000 \mathrm{HS} A$, Malvern Instruments, Malvern, UK).

The cantilever spring constant $\left(k_{\mathrm{sp}}\right)$ was determined independently using the method of Cleveland ${ }^{23}$ and found to be equal to the manufacturer's reported value $\left(k_{\mathrm{sp}}\right.$ $=0.06 \mathrm{~N} \mathrm{~m}^{-1}$ ) (see Fig. 2). Force curve data were collected as the mineral surface approached, made contact with, and subsequently separated from the cantilevers supporting immobilized cells at rates between 600 and $1000 \mathrm{~nm} \mathrm{~s}^{-1}$ (chosen to mimic typical swimming velocities of motile bacterial cells) starting at a maximum separation distance of $500 \mathrm{~nm}$ and at a frequency of $1 \mathrm{~Hz}$. Although operating at such high rates to mimic cell swimming velocities may result in the cantilevers being subject to hydraulic dampening, we maintained consistent experimental conditions (including solution viscosity) throughout the experiments, thus any potential hydraulic dampening effects are consistent across the three surfaces investigated here. Measurements were made using each cantilever (with attached cells) on all three iron oxide crystal faces. A maximum loading force between 1 and $6 \mathrm{nN}$ (with an average $\sim 3.5 \mathrm{nN}$ ) was applied during each experiment to ensure contact between the cell and mineral surface. On each face, force curves were collected at randomly chosen positions to generate a large set of independent measurements. In addition, force measurements were also collected on each
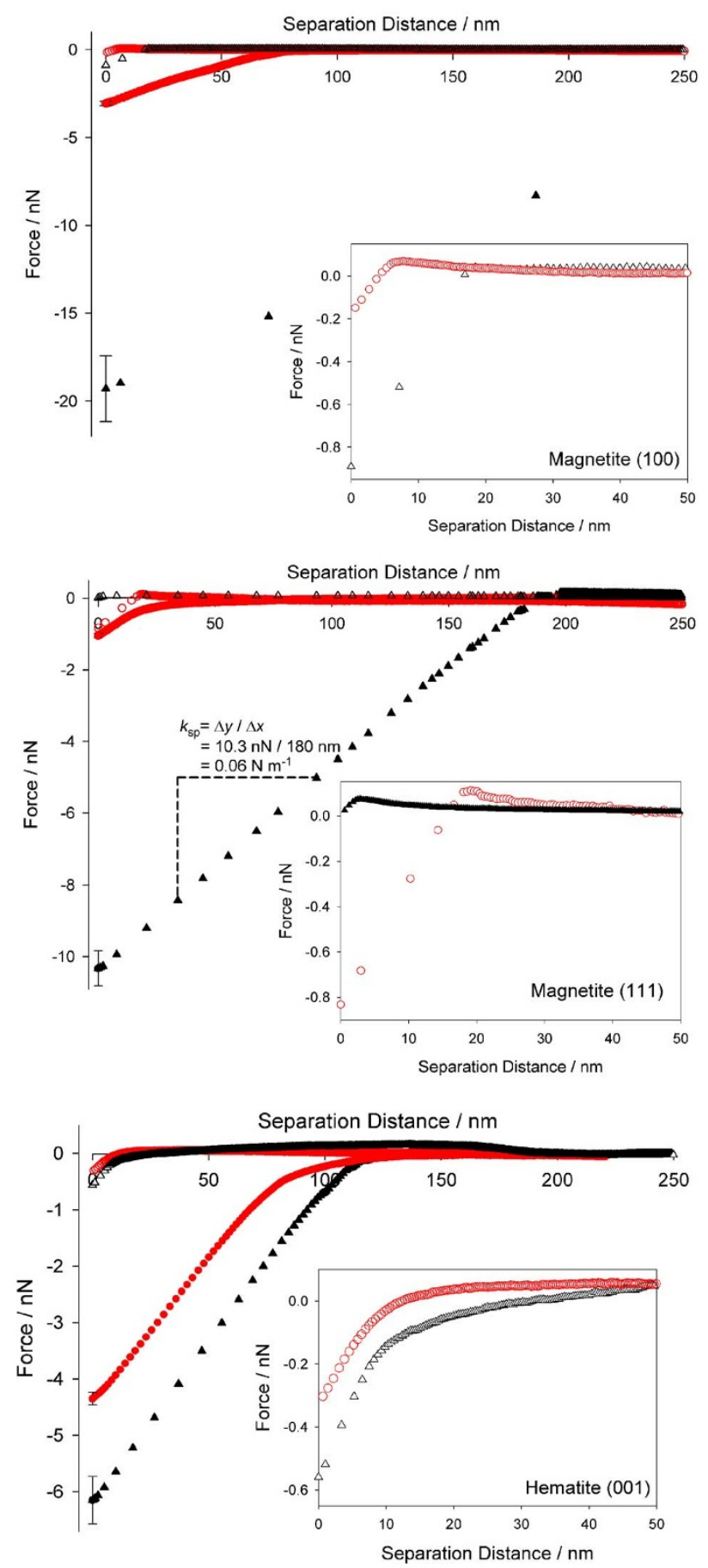

FIG. 2. (Color online) Interaction between 3-aminopropyltriethoxysilane functionalized cantilevers $(\boldsymbol{\Delta})$ and Shewanella-functionalized cantilevers (๑) on magnetite (100), magnetite (111), and hematite (001) crystal faces. Mean force-separation curves are shown. Approach curves are shown as open symbols, retraction curves as closed symbols, negative values represent attractive forces. Error bars for retraction curves at $0 \mathrm{~nm}$ separation represent 95\% Bonferroni confidence intervals of the mean force of adhesion. All measurements were made at $\mathrm{pH} 7$ in a PIPES-buffered basal medium. Cantilever spring constant $\left(k_{\mathrm{sp}}\right)$ was calculated at $0.06 \mathrm{~N} \mathrm{~m}^{-1}$ as shown. Insets show the forces generated upon approach at short separation distances.

mineral surface with APTES-functionalized cantilevers to which no cells had been attached. This treatment served to determine whether the forces measured were due to mineral- 
bacterial surface interactions or whether the APTES treated cantilevers exerted an influence which extended beyond the width of the attached cells $(\approx 250 \mathrm{~nm})$.

Data processing and analysis: Each collected force curve was analyzed separately. Those curves displaying significant noise due to the presence of gas bubbles in the liquid cell (evident as large plateaus), interference between light reflected from the cantilever and mineral surfaces (evident as periodic oscillations), or a steep positive or negative slope in the region of no contact were removed from the data set. The number of force curves collected on each mineral face ranged from 502 on Magnetite (100) to 901 on Magnetite (111), collected with a total of three cantilevers. Force data were collected as photodiode counts versus piezo displacement and converted to force versus cell-surface separation distance $^{24,25}$ using an IGOR-Pro 4.04 (WaveMetrics Inc., Lake Oswego, OR) routine, AFM 4.4 developed by Kendall and Hochella. ${ }^{26}$ This routine generates parameters for the forces of adhesion and repulsion (expressed in $\mathrm{nN}$ ) as well as jump-to-contact and jump-from-contact distances (expressed in $\mathrm{nm}$ ). The combined data sets were first analyzed collectively with a multiple analysis of variance (MANOVA). The effects of the different surfaces upon force curve parameters were subsequently analyzed by individual two-factor analysis of variance (ANOVA) using individual cantilevers and surfaces as factors. A priori, only those differences between crystal faces were of direct interest to the present study since it has previously been demonstrated that differences in adhesive properties even between clones of a single cell are to be expected. ${ }^{27,28}$ Therefore, in the case where a significant difference in force curve parameters was indicated by ANOVA $(\alpha=95 \%)$, Bonferroni pairwise comparisons were performed between the three surfaces.

\section{RESULTS}

\section{APTES-functionalized cantilevers}

Average force distance curves collected on approach to and retraction from the three mineral faces with APTESfunctionalized tipless cantilevers are shown in Fig. 2. Here we follow convention by ascribing positive signs to repulsive forces and negative signs to attractive forces. Little repulsive force was measured for APTES-functionalized cantilevers to which no cells were attached. Approach force-distance curves indicate little interaction between the APTES-treated cantilevers and the three mineral faces and that what interactions are observed occur only within separation distances of less than $20 \mathrm{~nm}$ (cf. immobilized cells below). Large forces of adhesion were observed upon retraction and are likely a function of the large amount of reactive surface area available on a tipless cantilever with no attached cell. Greatest forces (mean $\pm 95 \%$ confidence interval of the mean) were measured between APTES-functionalized cantilevers and magnetite $(100)(-19.3 \pm 0.7 \mathrm{nN})$. The smallest adhesive forces were generated between APTES-functionalized cantilevers and the hematite $(001)$ surface $(-6.2 \pm 0.2 \mathrm{nN})$. Intermediate forces were measured on the magnetite (111) surface $(-10.3 \pm 0.2 \mathrm{nN})$. An analysis of variance indicated a significant difference between the adhesive forces generated be-

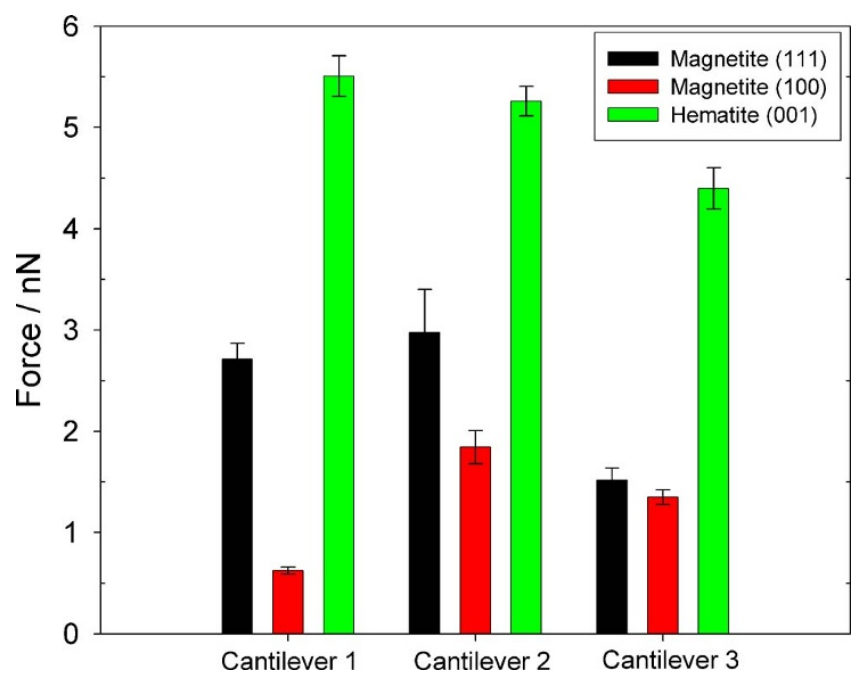

FIG. 3. (Color online) Mean force of adhesion upon retraction of Shewanella oneidensis MR-1 immobilized on APTES coated cantilevers from iron oxide single crystal faces. All forces are attractive. Error bars represent 95\% Bonferroni confidence intervals of the means. All measurements were made at $\mathrm{pH} 7$ in a PIPES-buffered basal medium.

tween the functionalized cantilever and the three surfaces $\left(\mathrm{F}_{2,171}=501.29, p<0.0005\right)$. Fisher post-test comparisons indicated significant $(\alpha=95 \%)$ differences between all three surfaces.

\section{Shewanella immobilized at the cantilever surface}

Cantilevers to which cells were attached displayed distinctly different interaction with the iron oxide surfaces than the APTES-functionalized cantilevers alone (Fig. 2). On approach, the attractive interactions between the APTESfunctionalized cantilevers and the three mineral faces are greater than those between the mineral faces and cantilevers with immobilized cells. On both magnetite surfaces the net effect of the cells is to lessen the attractive regime so that small repulsive forces are measurable at very short distances of separation. Greatest repulsive force is observed on the magnetite (111) surface with less repulsion observed on the magnetite (100). The bacterium-hematite (001) system, in contrast, exhibits no repulsive maximum upon approach, but the attractive regime is somewhat reduced in the presence of cells compared to forces measured using the ATPES functionalized cantilever. It is important to note that due to the size of these cells and the orientation of the cantilever, it is possible that the forces measured on approach are not due to the cells alone. The differences measured using functionalized cantilevers and cantilevers with attached cells however, are indeed due to cell-mineral interactions and not due to APTES-mineral interactions.

On retraction, clear qualitative and quantitative differences are observed in force curves between the three mineral surfaces and between the functionalized cantilevers and cantilevers with attached cells (Fig. 2). These differences are considered in greater detail below. Average adhesive forces collected with each cantilever on retraction are shown in Fig. 3. A separate study of the effects of loading force used in these experiments was also performed (data not shown). The results indicate that average adhesion forces measured be- 
tween immobilized cells and the iron oxide surfaces upon retraction vary only slightly $(<2 \mathrm{nN})$ as the maximum load force varies from $\sim 2$ to $4 \mathrm{nN}$. This indicates that even under very low loading force, measured forces derive largely from the immobilized cells. If APTES-functionalized cantilevers were to dominate the observed forces of adhesion, then at low loading force the adhesion forces measured upon retraction would be comparable to those measured employing the APTES-functionalized cantilevers with no immobilized cells. A multiple analysis of variance indicated significant differences between individual cantilevers (Wilks' $\lambda=0.873$, $\mathrm{F}_{2,2025}=147.95, p<0.0005$ ), as well as surfaces (Wilks' $\lambda$ $\left.=0.196, \mathrm{~F}_{2,2025}=4157.23, p<0.0005\right)$.

Interfacial forces upon approach: The mean $( \pm$ the $95 \%$ confidence interval of the mean) forces generated when the individual cantilevers and iron surfaces make contact for each system were $-0.16 \pm 0.02 \mathrm{nN}$ for the bacteriummagnetite (100) system, $-0.03 \pm 0.003 \mathrm{nN}$ for the bacteriummagnetite (111) system and $-0.40 \pm 0.02 \mathrm{nN}$ for the bacterium-hematite (001) system. Analysis of variance of the measured forces upon approach indicated a significant effect of both the cantilever $\left(\mathrm{F}_{2,2021}=200.65 ; p<0.0005\right)$ and surface $\left(\mathrm{F}_{2,2021}=400.20 ; p<0.0005\right)$ variables. There was also a significant interaction term $\left(\mathrm{F}_{4,2021}=120.30 ; p<0.0005\right)$. Upon inspection of the data for individual cells, this interaction term was found to arise from the fact that one of the three cell-functionalized cantilevers did not exhibit as great a force on the magnetite (111) face as either of the other two cantilevers. In every other respect, relative forces measured were consistent with the other two cantilevers. Pairwise comparisons between the individual surfaces indicated a significant difference between the two magnetite faces $(t=5.58 ; p$ $<0.0005)$ and between the magnetite (100) and hematite (001) faces $(t=12.93 ; p<0.0005)$.

Interfacial forces upon retraction: Mean forces of adhesion $( \pm 95 \%$ Bonferroni confidence interval of the mean) for each cantilever-mineral combination are shown in Fig. 3. Post-ANOVA Bonferroni comparisons of the mean forces generated upon retraction between APTES-functionalized cantilevers to which no cells were attached, and cellfunctionalized cantilevers indicated a maximum, significant difference $(\Delta)$ of $17.3 \mathrm{nN}(t=92.1 ; p<0.0005)$ for the magnetite (100) surface, for the magnetite (111) surface $\Delta$ $=9.3 \mathrm{nN}(t=67.4 ; p<0.0005)$ and the least difference between the two cantilever treatments is observed on hematite (001) $(\Delta=1.9 \mathrm{nN}, t=8.97 ; p<0.0005)$.

Despite expected differences between cantilevers (which include the number and position of attached cells as well as potential metabolic differences between those cells) there is a consistent trend in the data collected with different cantilevers on the three mineral faces. Bonferroni $95 \%$ confidence intervals suggest that for all cantilevers, significant differences are observed between the three mineral faces with the exception of the two magnetite faces measured with the third cantilever. Despite this, greatest forces of adhesion are consistently observed on the (001) hematite face, least force of adhesion is observed on the (111) magnetite face. The mean forces generated by each system across the three cantilevers upon retraction were $-2.0 \pm 0.04 \mathrm{nN}$ for the bacterium- magnetite (100) system, $-1.1 \pm 0.02 \mathrm{nN}$ for the bacteriummagnetite (111), and $-4.3 \pm 0.04 \mathrm{nN}$ for the bacteriumhematite (001) system. As for the approach curves, significant effects were observed for both cantilever $\left(\mathrm{F}_{2,2021}\right.$ $=190.67 ; p<0.0005)$ and surface $\left(\mathrm{F}_{2,2021}=4979.79 ; p\right.$ $<0.0005)$. Again there was a significant interaction $\left(F_{4,2021}\right.$ $=181.68 ; p<0.0005$ ), attributed in part to a lack of significant difference in the forces of adhesion measured for the two magnetite surfaces with cantilever 3 (see Fig. 3). In every other respect adhesion forces measured with cantilever 3 were consistent with the other two cantilevers. Pairwise comparisons between the means across the three cantilevers for individual surfaces indicated a significant difference between the two magnetite faces $(t=8.13 ; p<0.0005)$, between the magnetite (100) and hematite (001) faces $(t=18.30 ; p$ $<0.0005)$ and between (111) magnetite and (001) hematite $(t=13.47 ; p<0.0005)$.

\section{DISCUSSION}

In anaerobic environments, attachment to an iron oxide surface represents a significant event in the life history of iron reducing bacteria. Metabolism may be significantly impaired if the cell remains unattached or attaches to mineral surfaces lacking in Fe(III). Our limited knowledge of the cell surface of iron reducing bacteria such as Shewanella demonstrates properties suggesting intimate association with mineral surfaces is beneficial: lipopolysaccharides (LPS) of $S$. oneidensis MR-1 lack a polysaccharidic $O$-antigen side chain, ${ }^{29,30}$ and metal-reductases are positioned in the outer membrane. $^{31-33}$ The presence of $O$-antigen can significantly affect cell adhesion by masking membrane-associated functional groups; ${ }^{34,35}$ cells lacking $O$-antigen are better able to attach to surfaces possibly due to an increased exposure of the lipid moiety, thereby facilitating hydrophobic interactions $^{27,35,36}$ which is consistent with the implication of hydrophobicity in the attachment of $S$. algae to iron oxides. ${ }^{11}$ Positioning of metal-reductases in the outer membrane would allow the transfer of electrons from the inner membrane across the periplasm and then across the outer membrane to insoluble $\mathrm{Fe}$ (III) as the terminal electron acceptor. Electrostatic interactions have also been implicated in cell interactions with metals. ${ }^{37,38}$ At circum-neutral $\mathrm{pH}$ these are dominated by $\mathrm{COO}^{-}$groups $\left(p K_{\mathrm{a}} \approx 5\right)$ possibly associated with the LPS, but also with proteins.

We present data indicating that adhesive forces generated between Shewanella oneidensis cells and iron oxides reflect substratum mineralogy. Although we cannot wholly discount the possibility of the APTES coating contributing to the observed cell-derived forces, greatest forces of adhesion are observed between cells and a hematite (001) surface; differences are also observed between magnetite faces with the (111) face supporting greater adhesive forces than a (100) face. This series in adhesive force is consistent with previous observations of cell accumulation at the mineral faces where maximum cell densities are observed on hematite (001). ${ }^{10}$ Also, relatively more daughter cells are released from the two magnetite surfaces compared to hematite ${ }^{10}$ reflecting the reduced adhesive forces observed here. There is therefore 


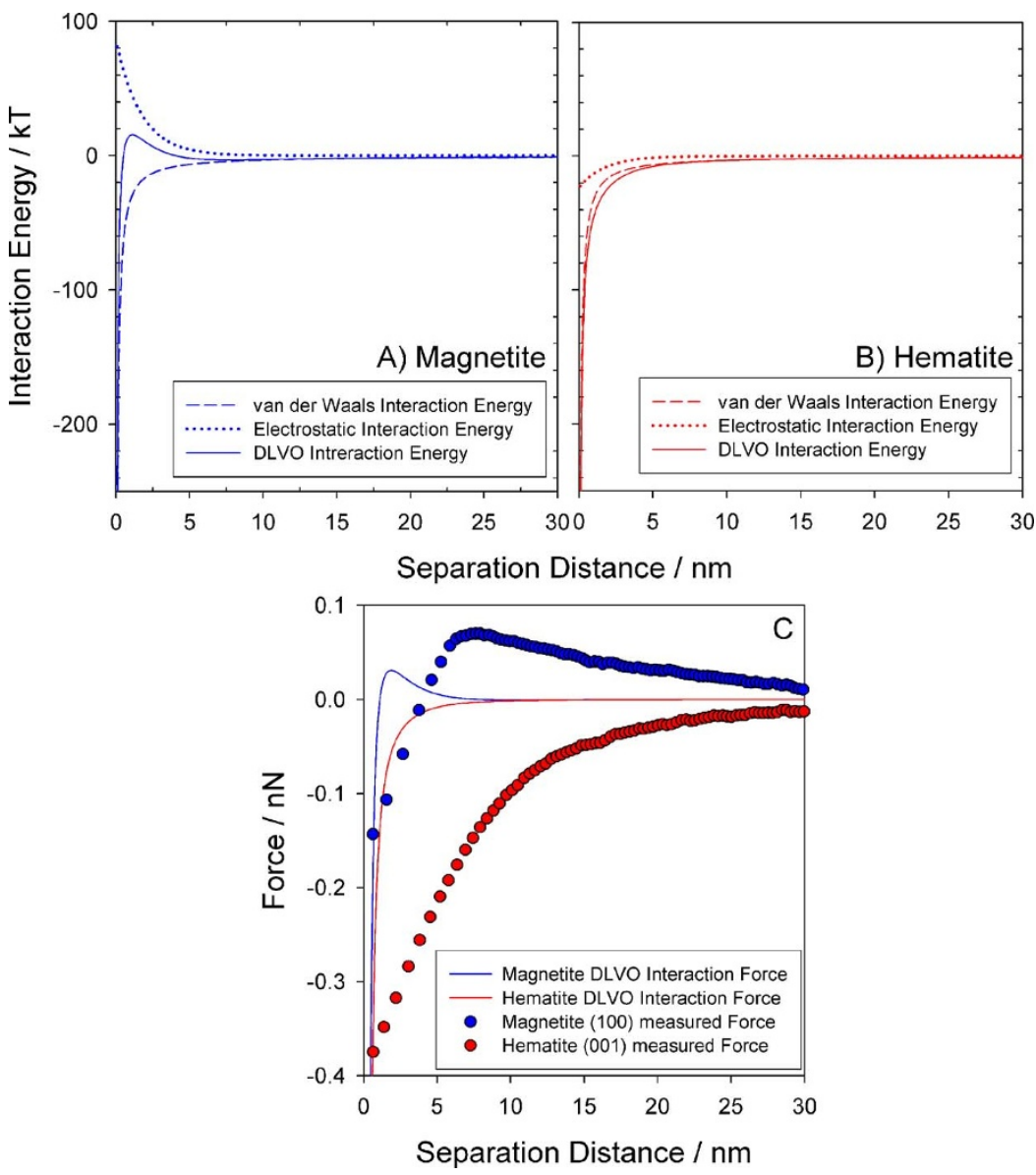

FIG. 4. (Color online) Classical DLVO predictions of interaction energy between $S$. oneidensis MR-1 cells and generalized (A) magnetite and (B) hematite surfaces. (C) Approach curves, recorded using atomic force microscopy employing MR-1 cells immobilized on APTES functionalized $\mathrm{Si}_{3} \mathrm{~N}_{4}$ cantilevers, to magnetite and hematite surfaces. A Hamaker constant of $3.22 \times 10^{-21} \mathrm{Nm}$ was assumed (Ref. 47 ), $\zeta$ potentialceLL was measured at $-5.7 \mathrm{mV}$ in $32 \mathrm{mM}$ PIPES buffer $(\mathrm{pH}$ 7.2); $\zeta$ potentialmineral was based upon $p \mathrm{H}_{\mathrm{PZC}}$ of $6.6 \pm 0.4$ and $8.3 \pm 0.9$ for magnetite and hematite, respectively (Ref. 40). DLVO calculations were performed for an ionic strength of $32 \mathrm{mM}$. Energy-to-force conversions were calculated as described by Cail and Hochella (Ref. 44).

compelling evidence to suggest that some, as yet unidentified, property of the mineral surface can influence not only adhesion/attachment but also growth and detachment of $S$. oneidensis cells. Predictions of the rate of electron transfer (ET) from OmcA, an outer membrane $c$-type cytochrome, to the respective mineral faces can be related to the observations of adhesive force: those surfaces to which ET is likely to be fastest, i.e., hematite (001), support the greatest force of adhesion and the greatest cell density.

Nonspecific adhesion, sensu Bos et al., ${ }^{39}$ is typically dominated by electrostatic forces and acid-base interactions. At circum-neutral $\mathrm{pH}, \mathrm{S}$. oneidensis cells have a net negative charge due largely to $\mathrm{COO}^{-}$groups associated with the outer membrane. ${ }^{37}$ We might assume therefore that the negatively charged cells $(\zeta$ potential CELLS $=-5.7 \mathrm{mV})$ will adhere with greater force to increasingly positively charged mineral surfaces; the observed series may therefore simply reflect surface charge of the three minerals. While nonface specific points of zero charge ( $\mathrm{pHPZC}$ ) for bulk mineral powders are available from acid-base titration data, few values are available for individual mineral faces. Moreover, reported values for bulk powders from different research groups are often not in close agreement due to differences in sample preparation, temperature, solution chemistry, and microscopic details such as the presence of defects and/or adsorbates. For bulk magnetite, a pHPZC of $6.6 \pm 0.4$ is generally accepted and for bulk hematite it is $8.3 \pm 0.9 .^{40}$ A single reported value specific to the hematite (001) face used here $\approx 8.5$ (Ref. 41) is consistent with reported bulk values. From the available pHpzc data, it is reasonable to assume only that under our experimental conditions $(\mathrm{pH} 7)$ the hematite face will be positively charged whilst the magnetite faces may be slightly negatively charged. This is consistent with the observed repulsive forces upon approach to the negatively charged magnetite surfaces (see Fig. 3) and the lack of any repulsive force generated between the cantilever-immobilized cells and the hematite (001) surface. In this context, it should be noted that the forces of interaction generated between MR-1 cells and magnetite and hematite surfaces calculated employing classical DLVO theory ${ }^{42}$ qualitatively resemble profiles measured by AFM (see Fig. 4). Thus, the series in interaction forces upon approach, hematite (001) > magnetite (100) $>$ magnetite (111), may in large part be due to electrostatic interactions. However, the magnitudes of the forces calculated by DLVO differ significantly at small separation distances compared to values measured by AFM. Previous research has suggested that DLVO theory fails to accurately predict force curves at very small distances of separation. ${ }^{43,44}$

Lower et al. ${ }^{17}$ suggest that $\mathrm{Fe}(\mathrm{III})$ at the surface of goethite may specifically increase adhesion of Shewanella compared to diaspore although this effect, increased cell attachment in the presence of surface associated iron, has itself previously been interpreted as the result of increased electrostatic interactions. ${ }^{45}$ Predicted ferric site density for the iron oxide faces considered here increases from $1.4 \mathrm{~nm}^{-2}$ on magnetite (100) to $4.2 \mathrm{~nm}^{-2}$ for hematite (001) with magnetite (111) having an intermediate density of $2.8 \mathrm{~nm}^{-2} .{ }^{10}$ Thus, ferric site density, and by extension surface charge, agree 
well with measurements of cell adhesion. The fact that we do not observe retraction curve features similar to those observed by Lower et al., on goethite also suggests that the Shewanella-iron oxide surface interaction measured in our experiments is not dominated by specific interactions inferred by Lower et al. ${ }^{18}$ It is pertinent to acknowledge at this point that there are a number of experimental differences between those described here and those described earlier by Lower et al.: ${ }^{18}$ cells were immobilized on a glass bead by Lower et al., although we do have more than a single cell immobilized on cantilever surfaces it is likely that the experimental surface areas differ when cells are immobilized at a flat, rather than a spherical surface; also we have employed single crystal faces in an attempt to minimize the effect of surface discontinuities upon the measured forces of adhesion and simple monotonic retraction curves are more typical of cell interactions with flat surfaces, including Escherichia coli D21 with variously functionalized glass cover slips ${ }^{36}$ and Burkholderia sp. with freshly cleaved muscovite. ${ }^{17}$ If sawtooth features exist in our retraction curves, it is possible they are masked by the large adhesive forces present in our system. It should be noted however that such sawtooth features are not observed in Lower et al. on diaspore surfaces. Finally, sawtooth features in retraction curves are observed only infrequently (no more than $10 \%$ of retraction curves display features ${ }^{46}$ ) thus we may not have collected a sufficient number of retraction curves to observe this stochastic feature.

The exact mechanism by which dissimilatory metal reducing bacteria attach to iron oxide mineral surfaces remains elusive. It is clear however that differences in the force of cell adhesion of Shewanella oneidensis are observed dependent upon the presence of ferric iron at mineral surfaces ${ }^{18}$ as well as between different iron oxide crystal faces (data presented here). Circumstantial evidence suggests that both electrostatic and hydrophobic interactions are important factors in determining not only the magnitude of adhesion force, but also the attachment behavior and growth of cell populations. ${ }^{10-12}$ It is not clear whether the apparent effect of ferric site density observed here operates via an exclusively electrostatic mechanism or whether some specific recognition of ferric iron is responsible. Since ferric reductases (in the form of cytochromes) are present in the outer membrane, it is tempting to describe cell attachment in terms of specific interactions (sensu Lower et al. ${ }^{18}$ ), however this hypothesis remains to be thoroughly tested.

\section{ACKNOWLEDGMENTS}

This research was supported by the Environmental Remediation Sciences Division (ERSD) of the Office of Biological and Environmental Research (OBER), U.S. Department of Energy, through Financial Assistance Grant No. DEFC09-96-SR18546 to the University of Georgia Research Foundation. The authors are also grateful to ERSD for financial support through Grants Nos. DE-FG02-02ER15326 and DE-FG02-05ER64121, and to the National Science Foundation through Grant No. EAR 01-03053. A portion of the research described in this paper was performed at the W. R.
Wiley Environmental Molecular Sciences Laboratory, a national scientific user facility also sponsored by OBER located at Pacific Northwest National Laboratory, operated for the Department of Energy by Battelle.

${ }^{1}$ E. E. Roden and J. M. Zachara, Environ. Sci. Technol. 30, 1618 (1996). ${ }^{2}$ D. R. Lovley and E. J. P. Phillips, Appl. Environ. Microbiol. 52, 751 (1986).

${ }^{3}$ E. E. Roden and R. G. Wetzel, Limnol. Oceanogr. 47, 198 (2002).

${ }^{4}$ E. E. Roden, Environ. Sci. Technol. 37, 1319 (2003).

${ }^{5}$ R. G. Arnold, T. J. DiChristina, and M. R. Hoffman, Biotechnol. Bioeng. 32, 1081 (1988).

${ }^{6}$ D. R. Lovley and E. J. P. Phillips, Appl. Environ. Microbiol. 54, 1472 (1988).

${ }^{7}$ A. Das and F. Caccavo, Curr. Microbiol. 40, 344 (2000).

${ }^{8}$ E. E. Roden and M. M. Urrutia, Environ. Sci. Technol. 33, 1847 (1999). ${ }^{9}$ E. E. Roden and M. M. Urrutia, Geomicrobiol. J. 19, 209 (2002).

${ }^{10}$ A. L. Neal, K. M. Rosso, G. G. Geesey, Y. A. Gorby, and B. J. Little, Geochim. Cosmochim. Acta 67, 4499 (2003).

${ }^{11}$ F. Caccavo, P. C. Schamberger, K. Keiding, and P. H. Nielsen, Appl. Environ. Microbiol. 63, 3837 (1997).

${ }^{12}$ F. Caccavo, Appl. Environ. Microbiol. 65, 5017 (1999).

${ }^{13}$ A. Danielsson, B. Norkrans, and A. Björnsson, Botan. Mar. 20, 13 (1977).

${ }^{14}$ S. Kjelleberg, "Adhesion to inanimate surfaces," Microbial Adhesion and Aggregation, edited by K. C. Marshall (Springer-Verlag, Berlin, 1984), pp. 51-70.

${ }^{15}$ T. A. Stenström, Appl. Environ. Microbiol. 55, 142 (1989).

${ }^{16}$ S. K. Lower, C. J. Tadanier, and M. F. Hochella, Geochim. Cosmochim. Acta 64, 3133 (2000).

${ }^{17}$ S. K. Lower, C. J. Tadanier, and M. F. Hochella, Geomicrobiol. J. 18, 63 (2001).

${ }^{18}$ S. K. Lower, M. F. Hochella, and T. J. Beveridge, Science 292, 1360 (2001).

${ }^{19}$ K. A. Edwards and A. D. Rutenberg, Chem. Geol. 190, 19 (2001).

${ }^{20}$ R. D. Boyd, J. Verran, M. V. Jones, and M. Bhakoo, Langmuir 18, 2343 (2002).

${ }^{21}$ J. E. Kostka and K. H. Nealson, "Isolation, cultivation, and characterisation of iron- and manganese-reducing bacteria," in Techniques in Microbial Ecology, edited by R. S. Burlage, R. Atlas, D. Stahl, G. G. Geesey, and G. Sayler (Oxford University Press, Oxford, 1988), pp. 58-78.

${ }^{22}$ A. S. Beliaev, D. M. Klingemann, J. A. Klappenbach, L. Wu, M. F. Romine, J. M. Tiedje, K. H. Nealson, J. K. Fredrickson, and J. Zhou, J. Bacteriol. 187, 7138 (2005).

${ }^{23}$ J. P. Cleveland, S. Manne, D. Bocek, and P. K. Hansma, Rev. Sci. Instrum. 64, 403 (1993).

${ }^{24}$ B. Capella and G. Dietler, Surf. Sci. Rep. 34, 1 (1999).

${ }^{25}$ Y. F. Dufrêne, J. Bacteriol. 184, 5205 (2002).

${ }^{26}$ T. A. Kendall and M. F. Hochella, Geochim. Cosmochim. Acta 67, 3537 (2003).

${ }^{27}$ V. Williams and M. Fletcher, Appl. Environ. Microbiol. 62, 100 (1996).

${ }^{28}$ J. C. Baygents, J. R. Glynn, O. Albinger, B. K. Biesemeyer, K. L. Ogden, and R. G. Arnold, Environ. Sci. Technol. 32, 1596 (1998).

${ }^{29}$ A. A. Korenevsky, E. Vinogradov, Y. Gorby, and T. J. Beveridge, Appl. Environ. Microbiol. 68, 4653 (2002).

${ }^{30}$ E. Vinogradov, A. Korenevsky, and T. J. Beveridge, Carbohydr. Res. 338, 1991 (2003).

${ }^{31}$ C. R. Myers and J. M. Myers, J. Bacteriol. 174, 3429 (1992).

${ }^{32}$ C. R. Myers and J. M. Myers, Biochim. Biophys. Acta 1326, 307 (1997).

${ }^{33}$ K. E. Pitts, P. S. Dobbin, F. Reyes-Ramirez, A. J. Thomson, D. J. Richardson, and H. E. Seward, J. Biol. Chem. 278, 27758 (2003).

${ }^{34}$ S. A. Makin and T. J. Beveridge, Microbiology 142, 299 (1996).

${ }^{35}$ M. Hermansson, S. Kjelleberg, T. K. Korhonen, and T. A. Stenström, Arch. Microbiol. 131, 308 (1982).

${ }^{36}$ A. Razatos, Y.-L. Ong, M. M. Sharma, and G. Georgiou, Proc. Natl. Acad. Sci. U.S.A. 95, 11059 (1998).

${ }^{37}$ J. R. Haas, T. J. Dichristina, and R. Wade, Chem. Geol. 180, 33 (2001).

${ }^{38}$ D. S. Smith and F. G. Ferris, J. Colloid Interface Sci. 266, 60 (2003).

${ }^{39}$ R. Bos, H. C. van der Mei, and H. J. Busscher, FEMS Microbiol. Lett. 23, 179-230 (1999).

${ }^{40}$ R. M. Cornell and U. Schwertmann, The Iron Oxides: Structure, Properties, Reactions, Occurrence and Uses (VCH, New York, 1996).

${ }^{41}$ C. M. Eggleston and G. Jordan, Geochim. Cosmochim. Acta 62, 1919 (1998). 
${ }^{42}$ C. J. van Oss, Interfacial Forces in Aqueous Media (Marcel Dekker, New York, 1994).

${ }^{43}$ J. A. Brant and A. E. Childress, Environ. Eng. Sci. 19, 413 (2002).

${ }^{44}$ T. L. Cail and M. F. Hochella, Environ. Sci. Technol. 39, 1011 (2005).

${ }^{45}$ A. L. Mills, J. S. Herman, G. M. Hornberger, and T. H. De Jesús, Appl.
Environ. Microbiol. 60, 3300 (1994).

${ }^{46}$ B. H. Lower, R. Yongsunthon, F. P. Vellano, and S. K. Lower, J. Bacteriol. 187, 2127 (2005).

${ }^{47}$ P. K. Sharma and K. Hanumantha Rao, Colloids Surf., B 24, 11 (2003). 